\title{
Electroconvulsive therapy does not alter the synaptic protein neurogranin in the cerebrospinal fluid of patients with major depression.
}

Laura Kranaster ${ }^{\text {a }}$, Carolin Hoyer ${ }^{\text {b }}$, Suna Su Aksay ${ }^{a}$, Jan Malte Bumb c , Christoph Janke ${ }^{d}$, Manfred Thiel d, Kaj Blennow ${ }^{e, f}$, Henrik Zetterberg e,f,g,h, Alexander Sartorius ${ }^{*}$

a Department of Psychiatry and Psychotherapy, Central Institute of Mental Health, Medical Faculty Mannheim / Heidelberg University, Mannheim, Germany

${ }^{b}$ Department of Neurology, University Medical Centre Mannheim, Mannheim, Germany

${ }^{c}$ Department of Addictive Behavior and Addiction Medicine, Central Institute of Mental Health, Medical Faculty Mannheim/Heidelberg University, Mannheim, Germany

d Department of Anesthesiology and Critical Care Medicine, University Medical Centre Mannheim, Mannheim, Germany

e Department of Psychiatry and Neurochemistry, Institute of Neuroscience and Physiology, the Sahlgrenska Academy at the University of Gothenburg, Mölndal, Sweden

${ }^{\dagger}$ Clinical Neurochemistry Laboratory, Sahlgrenska University Hospital, Mölndal, Sweden

${ }^{g}$ Department of Molecular Neuroscience, UCL Institute of Neurology, London, UK

h UK Dementia Research Institute at UCL, London, UK.

\section{Corresponding author}

Laura Kranaster

Department of Psychiatry and Psychotherapy

Central Institute of Mental Health, Medical Faculty Mannheim / Heidelberg University J5, 68159 Mannheim, Germany

Phone: +49 621 1703-0 - Fax: +49621 1703-1205 - Mail: laura.kranaster@zi-mannheim.de ORCID: 0000-0002-8414-0910

\section{Acknowledgments}

LK received support by the German Research Foundation (DFG - grant No. KR 4689/3-1). 


\section{Abstract}

Neurogranin $(\mathrm{Ng})$ is a novel marker proposed to measure neurodegeneration in Alzheimer's disease and to be associated with synaptic plasticity. It was tested whether a course of electroconvulsive therapy (ECT) could modify CSF Ng concentration. CSF Ng concentrations were measured before and after a course of ECT in the cerebrospinal fluid in twelve patients with major depression. $\mathrm{Ng}$ concentrations did not change during ECT, but baseline levels were positively correlated with the therapeutic response, which suggests an involvement of $\mathrm{Ng}$ in depression.

\section{Keywords}

ECT, electroconvulsive therapy, neurogranin, depression, affective disorder, cerebrospinal fluid 


\section{Introduction}

Electroconvulsive therapy (ECT) is a highly effective, non-pharmacological treatment option for severe and treatment-resistant forms of depression, mania and schizophrenia. The complex mode of action of ECT has not yet been fully unravelled, but among other mechanism, it is considered to facilitate neurogenesis and neural plasticity (Inta et al. 2013; Rotheneichner et al. 2014; Sartorius et al. 2009). Recently, the interplay between antidepressant treatment and amyloid $\beta(A \beta)$ metabolism in humans was investigated by analyzing the antidepressant effects of ECT on A $\beta$ peptides in the cerebrospinal fluid (CSF) in patients with major depression. CSF levels of $A \beta_{1-42}$, one of the major key players in the pathogenesis of Alzheimer's disease $(A D)$, were elevated after the ECT treatment and an increase of $A \beta_{1-42}$ after ECT was found in all patients with clinical response to the ECT treatment, but not in those who did not respond (Kranaster et al. 2016). In contrast to this, $A \beta_{1-40}$ and tau protein and its abnormal phosphorylated isoform were not altered by ECT.

To complement the evaluation of the effect of ECT on proteins involved in neurodegeneration, neurogranin $(\mathrm{Ng})$, a novel marker, that has been suggested to measure neurodegeneration in $A D$ and is highly correlated with tau and $A \beta$ pathology in $A D$ (Mattsson et al. 2016) was analysed. $\mathrm{Ng}$ is a post-synaptic protein (Gerendasy and Sutcliffe 1997) and its CSF level is a putative marker of synaptic loss in AD and it is associated with brain atrophy (Portelius et al. 2015). $\mathrm{Ng}$ is expressed in the cortex and hippocampus by excitatory neurons (Represa et al. 1990; Guadano-Ferraz et al. 2005), has a key role in synaptic plasticity and induction of longterm potentiation (LTP) in the hippocampus (Fedorov et al. 1995; Chen et al. 1997) and could restore $A \beta$-mediated synaptic plasticity deficits (Kaleka and Gerges 2016).

Based on these findings, we aimed to test, whether the neuronal plasticity, which is known to be enhanced by ECT (Joshi et al. 2016) is mediated by Ng modulation that could be detected by changes of $\mathrm{Ng}$ levels in the CSF in patients who receive ECT due to their major depression. 


\section{Methods}

\section{Patients}

Our prospective study was approved by the ethics committee and was performed in accordance with The Code of Ethics of the World Medical Association (Declaration of Helsinki). Written informed consent was obtained from all participants prior to enrolment. The study took place from 2013 to 2015 at the Department of Psychiatry at the Central Institute of Mental Health in Mannheim, Germany. Inclusion criteria were a present depressive episode within the context of a diagnosis of either major depressive disorder or bipolar disorder according to DSM-IV, age above 18 years and the clinical decision for an ECT treatment. Exclusion criteria were substance-related disorders and a lifetime diagnosis of schizophrenia. The Hamilton Depression Rating Scale (HDRS; 21 items version) and Mini-Mental-State Examination (MMSE) were used to assess the severity of depression and cognitive performance, respectively, before and after the ECT in each patient.

\section{ECT treatment}

ECT was performed with a Thymatron IV device (Somatics, LLC. Lake Bluff, IL, USA) with 900 $\mathrm{mA}$ constant current and bidirectional brief pulse stimulation. S-ketamine was used as the anaesthetic substance $(\sim 1.0 \mathrm{mg} / \mathrm{kg}$ ) (Hoyer et al. 2014; Kranaster et al. 2011) and succinylcholine was used for muscle relaxation $(\sim 1.0 \mathrm{mg} / \mathrm{kg})$. Seizure threshold in all patients was titrated and stimulus dose at subsequent treatments was given at 2.5 times threshold. The charge was subsequently increased if patients did not respond clinically or if seizures were insufficient during the ECT course according to established recommendations (American Psychiatric Association 2001). Two or three ECT sessions per week were performed. All patients started with right unilateral stimulation, in the case of non-response it was possible to switch to bilateral stimulation as a decision of the ECT supervisor.

\section{Sampling}

Before the first ECT session and between one and seven days after the last ECT session, CSF samples were drawn at around 9:00 am. The patients were in a non-fasting status. They had 
a light breakfast at 8:00 am. Lumbar punctures were performed in the sitting position according to standardized procedure. CSF samples $(\mathrm{X} \mathrm{mL})$ were obtained, centrifuged (2000 $\mathrm{x} \mathrm{g}$ ?), aliquoted into $\mathrm{XXX} \mathrm{mL}$ portions that were frozen at $-80^{\circ} \mathrm{C}$ within $30 \mathrm{~min}$. The samples were stored at $-80^{\circ} \mathrm{C}$ and were never thawed or refrozen prior to $\mathrm{Ng}$ analysis.

\section{CSF laboratory analyses}

All samples were measured at the Clinical Neurochemistry Laboratory at Sahlgrenska University Hospital. CSF $\mathrm{Ng}$ concentration was measured by enzyme-linked immunosorbent assay (ELISA), essentially as described (http://www.ncbi.nlm.nih.gov/pubmed/25533203), using the anti- $\mathrm{Ng}$ antibody pair $\mathrm{Ng} 2$ and $\mathrm{Ng} 22$. Baseline and follow-up samples were analyzed on one occasion on the same plates using the same batch of reagents. Intra-assay coefficients of variation were below $10 \%$.

\section{Statistical analyses}

Statistics were performed using STATA® (StataCorp, Texas 77845, USA, version 11) at a significance level $\leq 0.05$. Non-parametric tests were used, because the $\mathrm{Ng}$ levels were not normally distributed. The impact of ECT on changes in the CSF $\mathrm{Ng}$ concentration was calculated with the Wilcoxon matched-pairs signed-ranks test. Correlations were tested with the Spearman's rank correlation. In the case of values below the level of detection (LOD), it was decided to substitute them with LOD/2, although we are aware of the potential bias and imprecision (Ogden 2010).

\section{Results}

We included twelve patients into the study, who underwent a completed course of ECT and lumbar puncture prior to and following a treatment course. In five samples, neurogranin levels were below the limit of detection of $40 \mathrm{pg} / \mathrm{ml}$ (in two patients in both samples and in another patient in the baseline sample). Demographic and clinical features are shown in Table 1.

\section{Differences before and after ECT}


CSF neurogranin concentration at baseline $(117.7 \mathrm{pg} / \mathrm{ml} \pm 107.0)$ and after the complete ECT treatment $(122.8 \mathrm{pg} / \mathrm{ml} \pm 80.4)$ did not differ from each other $(\mathrm{p}=0.15)$. The mean neurogranin baseline level in the group of those patients suffering from Alzheimer's disease (AD) was higher $(179.5 \mathrm{pg} / \mathrm{ml} \pm 77.8)$ at a trend level significance than the mean level of the patients, who did not suffer from $A D(97.1 \mathrm{pg} / \mathrm{ml} \pm 111.0 ; \mathrm{p}=0.077)$. The change in neurogranin levels during ECT did not differ in these two groups $(p=0.78)$.

\section{Correlation with numbers of ECT sessions}

The changes from baseline to final levels were not associated with the number of single ECT sessions that were performed in each patient $(p=0.74)$.

\section{Antidepressant efficacy}

The changes of neurogranin levels during ECT were neither associated with change of depressive psychopathology during ECT $(p=0.93)$, nor with therapeutic response $(p=0.26)$ or remission ( $p=0.94)$. The baseline level of CSF neurogranin was positively correlated with the reduction of depressive psychopathology during ECT (Figure $1 ; r_{s}=0.64 ; p=0.024$ ) and the therapeutic response $\left(r_{s}=0.59 ; p=0.045\right)$.

\section{Possible moderators}

In our sample, CSF neurogranin levels were not associated with age $(p=0.37)$, sex $(p=0.94)$, bipolarity $(p=0.93)$, education $(p=0.51)$ and MMSE at baseline $(p=0.90)$.

\section{Discussion}

This is the first study to investigate changes of Ng CSF levels in response to ECT treatment in patients suffering from depression. In contrast to the reported changes in the $A \beta$ metabolism during ECT (Kranaster et al. 2016), we could not detect an alteration of the synaptic protein $\mathrm{Ng}$ during ECT and no associated with the changes of CSF $\mathrm{Ng}$ levels and the number of individual ECT sessions. Thus, CSF Ng concentration does not seem to reflect the enhanced neuronal plasticity that has been observed after ECT (Joshi et al. 2016). 
As a secondary finding, the baseline level of CSF $\mathrm{Ng}$ was positively correlated with the reduction of depressive psychopathology and a better therapeutic response to ECT. Because the role of $\mathrm{Ng}$ in mood disorders has not been investigated in-depth yet, our data are the first concerning prediction of antidepressant treatment response based on CSF Ng levels. There is only one previous report, in which data on CSF $\mathrm{Ng}$ levels in patients with major depressive disorder were reported. Their cohort consisted only of six patients, but interestingly all of them had considerable low CSF Ng levels (Sanfilippo et al. 2016). Although measured by the same group, the research grade assay was modified in-between, which makes it impossible to set our data in perspective with the historical data to test whether the $\mathrm{Ng}$ concentration in our sample of depressive patients are also lower compared to age-matched healthy controls or to patients with neurodegenerative diseases. One very indirect hint, that the CSF Ng concentration in our patients can also be considered as considerable low is that in three out of twelve patients (25\%) at baseline the $\mathrm{Ng}$ levels were under the limit of detection. At least, no similar pattern was reported in previous cohorts of healthy controls (Sanfilippo et al. 2016; Portelius et al. 2015). Additionally, $\mathrm{Ng}$ has been recognized as a susceptibility gene of major depression and bipolar disorder (and schizophrenia) in Han Chinese (Wen et al. 2016). Based on these few data, it could be speculated, that $\mathrm{Ng}$ has indeed a role in the development and treatment of major depression and that it might be worthwhile to evaluate the potential interplay of $\mathrm{Ng}$ and depression in more detail.

The major limitation of this study is the small sample size, but the difficulty in recruiting severely depressed patients who agree to undergo lumbar puncture twice for research purposes only in addition to a course of ECT should not be underestimated. It could not be excluded, that the insufficient power lead to the negative primary finding. However, it was possible to detect changes in other systems with that small sample size (Kranaster et al. 2017b; Kranaster et al. 2017a) and at least a strong effect of ECT on $\mathrm{Ng}$ appears unlikely.

In conclusion, mean CSF Ng concentrations did not change during a course of ECT, but the apparently low baseline $\mathrm{Ng}$ levels in the patients with major depression were positively 
correlated the amount of therapeutic response, which suggests an involvement of $\mathrm{Ng}$ in depression.

\section{Conflict of interest}

$\mathrm{KB}$ and $\mathrm{HZ}$ are co-founders of Brain Biomarker Solutions in Gothenburg AB, a GU Venturesbased platform company at the University of Gothenburg. The other authors declare that they have no conflict of interest. 


\section{References}

American Psychiatric Association (2001) The Practice of Electroconvulsive Therapy: Recommendations for Treatment, Training, and Privileging (A Task Force Report of the American Psychiatric Association). 2nd edn. American Psychiatric Association, Washington, DC

Chen SJ, Sweatt JD, Klann E (1997) Enhanced phosphorylation of the postsynaptic protein kinase $\mathrm{C}$ substrate RC3/neurogranin during long-term potentiation. Brain Res 749 (2):181-187

Fedorov NB, Pasinelli P, Oestreicher AB, DeGraan PN, Reymann KG (1995) Antibodies to postsynaptic PKC substrate neurogranin prevent long-term potentiation in hippocampal CA1 neurons. The European journal of neuroscience 7 (4):819-822

Gerendasy DD, Sutcliffe JG (1997) RC3/neurogranin, a postsynaptic calpacitin for setting the response threshold to calcium influxes. Mol Neurobiol 15 (2):131-163

Guadano-Ferraz A, Vinuela A, Oeding G, Bernal J, Rausell E (2005) RC3/neurogranin is expressed in pyramidal neurons of motor and somatosensory cortex in normal and denervated monkeys. The Journal of comparative neurology 493 (4):554-570. doi:10.1002/cne.20774

Hoyer C, Kranaster L, Janke C, Sartorius A (2014) Impact of the anesthetic agents ketamine, etomidate, thiopental, and propofol on seizure parameters and seizure quality in electroconvulsive therapy: a retrospective study. Eur Arch Psychiatry Clin Neurosci 264 (3):255-261. doi:10.1007/s00406-013-0420-5

Inta D, Lima-Ojeda JM, Lau T, Tang W, Dormann C, Sprengel R, Schloss P, Sartorius A, Meyer-Lindenberg A, Gass P (2013) Electroconvulsive therapy induces neurogenesis in frontal rat brain areas. PLoS One 8 (7):e69869. doi:10.1371/journal.pone.0069869

Joshi SH, Espinoza RT, Pirnia T, Shi J, Wang Y, Ayers B, Leaver A, Woods RP, Narr KL (2016) Structural Plasticity of the Hippocampus and Amygdala Induced by Electroconvulsive Therapy in Major Depression. Biological psychiatry 79 (4):282-292. doi:10.1016/j.biopsych.2015.02.029

Kaleka KS, Gerges NZ (2016) Neurogranin restores amyloid beta-mediated synaptic transmission and long-term potentiation deficits. Exp Neurol 277:115-123. doi:10.1016/j.expneurol.2015.12.013

Kranaster L, Aksay SS, Bumb JM, Janke C, Alonso A, Hoyer C, Zerr I, Schmitz M, Hausner L, Frolich L, Sartorius A (2016) Electroconvulsive therapy selectively enhances amyloid beta 1-42 in the cerebrospinal fluid of patients with major depression: A prospective $\begin{array}{llll}\text { pilot study. Eur Neuropsychopharmacol } 26 & \text { (12):1877-1884. }\end{array}$ doi:10.1016/j.euroneuro.2016.11.004

Kranaster L, Hoyer C, Aksay SS, Bumb JM, Leweke FM, Janke C, Thiel M, Lutz B, Bindila L, Sartorius A (2017a) Electroconvulsive therapy enhances endocannabinoids in the cerebrospinal fluid of patients with major depression: a preliminary prospective study. Eur Arch Psychiatry Clin Neurosci. doi:10.1007/s00406-017-0789-7

Kranaster L, Hoyer C, Aksay SS, Bumb JM, Muller N, Zill P, Schwarz MJ, Sartorius A (2017b) Antidepressant efficacy of electroconvulsive therapy is associated with a reduction of the innate cellular immune activity in the cerebrospinal fluid in patients with depression. World J Biol Psychiatry:1-11. doi:10.1080/15622975.2017.1355473

Kranaster L, Kammerer-Ciernioch J, Hoyer C, Sartorius A (2011) Clinically favourable effects of ketamine as an anaesthetic for electroconvulsive therapy: a retrospective study. Eur Arch Psychiatry Clin Neurosci 261 (8):575-582. doi:10.1007/s00406-011-0205-7

Mattsson N, Insel PS, Palmqvist S, Portelius E, Zetterberg H, Weiner M, Blennow K, Hansson O, Alzheimer's Disease Neuroimaging I (2016) Cerebrospinal fluid tau, neurogranin, and neurofilament light in Alzheimer's disease. EMBO Mol Med 8 (10):1184-1196. doi:10.15252/emmm.201606540

Ogden TL (2010) Handling results below the level of detection. Ann Occup Hyg 54 (3):255256. doi:10.1093/annhyg/mep099 
Portelius E, Zetterberg H, Skillback T, Tornqvist U, Andreasson U, Trojanowski JQ, Weiner MW, Shaw LM, Mattsson N, Blennow K, Alzheimer's Disease Neuroimaging I (2015) Cerebrospinal fluid neurogranin: relation to cognition and neurodegeneration in Alzheimer's disease. Brain 138 (Pt 11):3373-3385. doi:10.1093/brain/awv267

Represa A, Deloulme JC, Sensenbrenner M, Ben-Ari Y, Baudier J (1990) Neurogranin: immunocytochemical localization of a brain-specific protein kinase $\mathrm{C}$ substrate. $\mathrm{J}$ Neurosci 10 (12):3782-3792

Rotheneichner P, Lange S, O'Sullivan A, Marschallinger J, Zaunmair P, Geretsegger C, Aigner L, Couillard-Despres S (2014) Hippocampal neurogenesis and antidepressive therapy: shocking relations. Neural Plast 2014:723915. doi:10.1155/2014/723915

Sanfilippo C, Forlenza O, Zetterberg H, Blennow K (2016) Increased neurogranin concentrations in cerebrospinal fluid of Alzheimer's disease and in mild cognitive impairment due to AD. J Neural Transm (Vienna) 123 (12):1443-1447. doi:10.1007/s00702-016-1597-3

Sartorius A, Hellweg R, Litzke J, Vogt M, Dormann C, Vollmayr B, Danker-Hopfe H, Gass P (2009) Correlations and discrepancies between serum and brain tissue levels of neurotrophins after electroconvulsive treatment in rats. Pharmacopsychiatry 42 (6):270-276. doi:10.1055/s-0029-1224162

Wen Z, Chen J, Khan RA, Wang M, Song Z, Li Z, Shen J, Li W, Shi Y (2016) Polymorphisms in NRGN are associated with schizophrenia, major depressive disorder and bipolar disorder in the Han Chinese population. Journal of affective disorders 194:180-187. doi:10.1016/j.jad.2016.01.034 


\section{Tables}

\begin{tabular}{|l|l|}
\hline Number of included subjects & 12 \\
\hline Age (mean) \pm SE in years (min - max) & $59.0 \pm 21.9(21-83)$ \\
\hline Sex female/male n/n (in \%) & $7 / 5(58.3 / 41.7)$ \\
\hline Type of depression: unipolar/bipolar n/n (in \%) & $9 / 3(75.0 / 25.0)$ \\
\hline HDRS, sum score & \\
\hline Baseline HDRS, mean \pm SE (min - max) & $29.9 \pm 6.6(21-41)$ \\
\hline Final HDRS, mean \pm SE (min - max) & $9.0 \pm 5.2(3-16)$ \\
\hline Mean change (min - max) & $-20.9(-6--36)(p<0.001)$ \\
\hline Numbers of ECT sessions, mean \pm SE (min - max) & $10.6 \pm 5.0(4-19)$ \\
\hline Switch to bilateral stimulation $n$ (in \%) & $3(25)$ \\
\hline Responders/Non-responders* $n / n($ in $\%)$ & $10 / 2(83.4 / 16.7)$ \\
\hline MMSE, sum score & \\
\hline Baseline MMSE, mean \pm SE (min - max) & $26.1 \pm 4.3(17-30)$ \\
\hline Final MMSE mean \pm SE (min - max) & $26.6 \pm 3.7(18-30)$ \\
\hline Mean change (min - max) & $0.6(-2-4)(p=0.30)$ \\
\hline
\end{tabular}

Table 1: Demographic and clinical features of the patients.

* Response is defined as a reduction of at least $50 \%$ in symptoms, measured by the HDRS. 


\section{Figures}

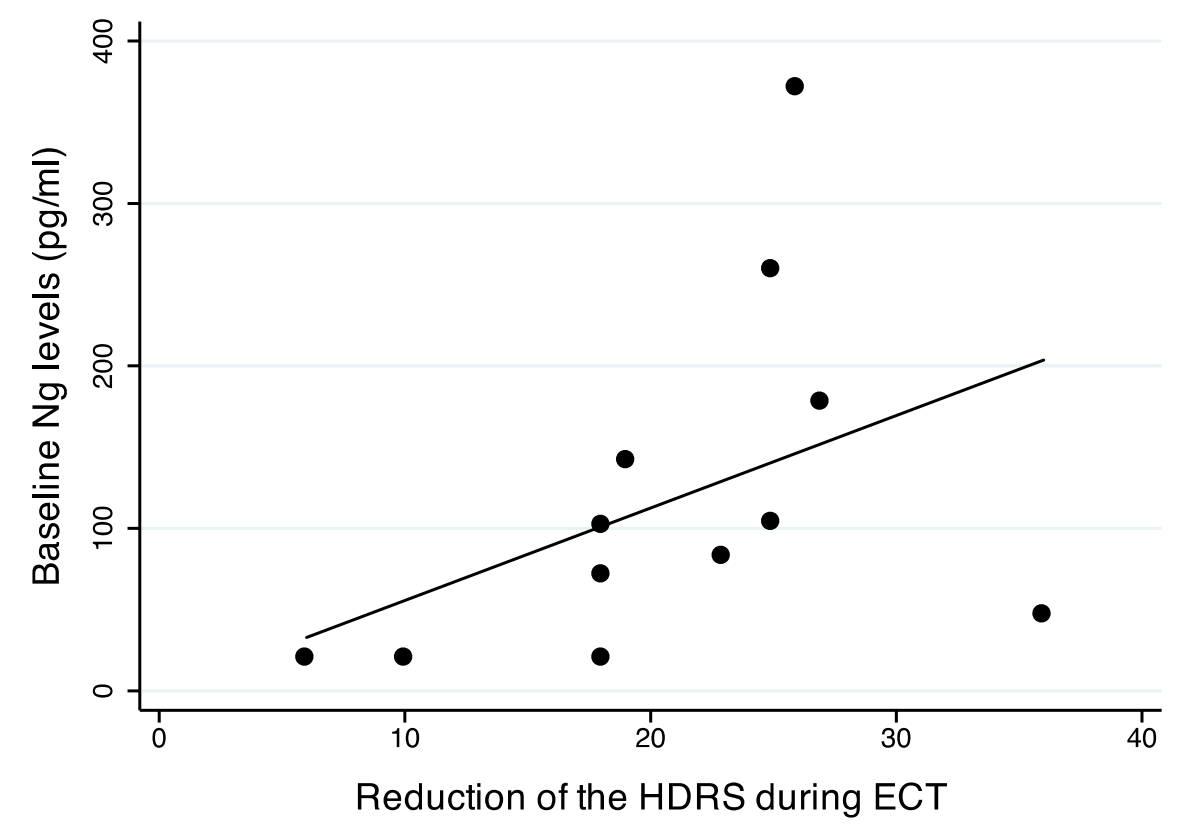

Figure 1: Correlation between CSF Ng levels at baseline and the reduction of the HDRS during $E C T\left(r_{s}=0.64 ; p=0.024\right)$. 